\title{
Autonomic dysreflexia
}

\author{
Jacquelyn Cragg MPH, Andrei Krassioukov MD PhD
}

\section{Autonomic dysreflexia is acute episodic hypertension resulting from sympa- thetic hyperactivity}

Autonomic dysreflexia occurs to some extent in up to $90 \%$ of people with upper thoracic (above the neurologic level of T6) and cervical spinal cord injury (Box 1). ${ }^{1}$ Although it can be asymptomatic, the signs and symptoms of autonomic dysreflexia generally include a sudden increase in blood pressure, altered heart rate (reflex bradycardia), anxiety, blurred vision, headache, flushing and sweating

\section{Box 1: Pathophysiology}

- Dysreflexia is related to the interruption of descending inhibition and the development of hyperresponsiveness of peripheral receptors.

- Bradycardia resulting from a hypertensive crisis is due to activation of baroreceptors and a vagal-mediated response above the lesion to compensate for the hypertensive crisis. (above the level of injury). ${ }^{1}$ Because people with upper thoracic and cervical injuries often have a low resting blood pressure (90$100 \mathrm{~mm} \mathrm{Hg}$ ), elevated blood pressure from autonomic dysreflexia may not always be obvious.
Autonomic dysreflexia is a medical emergency and can be life threatening

This condition has been linked with cardiovascular events such as myocardial ischemia and fatal cerebral hemorrhage. ${ }^{3,4}$ Unstable control of blood pressure (including autonomic dysreflexia), endothelial dysfunction, glucose intolerance and metabolic syndrome are the major contributors to early cardiovascularrelated complications and death in people with spinal cord injury.

\section{Guidelines recommend several steps to manage autonomic dysreflexia}

First, sit the patient upright to induce the orthostatic hypotension response. Second, loosen or remove any restrictive clothing and monitor the patient's heart rate and blood pressure at fiveminute intervals. Third, perform a survey of the possible causes of autonomic dysreflexia, starting with bladder distension. Lastly, if the patient's systolic blood pressure remains above $150 \mathrm{~mm} \mathrm{Hg}$, administer a short- and fast-acting antihypertensive agent, such as nifedipine or captopril. ${ }^{1}$

Competing interests: Andrei Krassioukov has recieved grants from Paralyzed Veterans of America and the Rick Hansen Institute. No competing interests declared by Jacquelyn Cragg.

This article has been peer reviewed.

Affiliation: From the International Collaboration on Repair Discoveries, University of British Columbia, Vancouver, BC

Correspondence to: Jacquelyn Cragg, craggj@interchange.ubc.ca

CMAJ 2012. DOI:10.1503/cmaj.110859
Autonomic dysreflexia is triggered by stimuli from below the level of injury

Autonomic dysreflexia can occur on a daily basis and can be triggered by stimuli such as distension of the bladder (most common), bladder or kidney stones, a kink in a urinary catheter, infection of the urinary tract, fecal impaction, pressure sores, an ingrown toenail, fractures, menstruation, hemorrhoids, invasive testing and sexual intercourse. ${ }^{1}$ Even benign stimuli, such as a shoelace that is tied too tightly or a sunburn can trigger autonomic dysreflexia. This condition usually resolves as soon as the precipitating stimuli are eliminated (e.g., bladder voided or colon emptied). In some cases, episodes do not resolve simply with removal of the stimulus. ${ }^{2}$

Awareness of the triggers of autonomic dysreflexia is the key to prevention

Appropriate management of bladder care and bowel routine is the best preventive measure because most episodes of autonomic dysreflexia are triggered by such stimuli. ${ }^{1}$ Prior consultation with a specialist in spinal cord injury before medical procedures should be considered to prevent iatrogenically induced autonomic dysreflexia.

For references, please see Appendix 1, available at www.cmaj.ca/lookup/suppl /doi:10.1503/cmaj.110859/-/DC1 